\title{
Isolement des constituants de l'arôme des fromages : comparaison de méthodes
}

\author{
par \\ J.P. DUMONT et J. ADDA \\ Laboratoire d'Etude des Arômes - I.N.R.A. - Jouy-en-Josas
}

Parmi les nombreux travaux portant sur l'étude des arômes des produits laitiers, il en est peu où soient exposées les raisons qui ont présidé au choix des méthodes utilisées. D'autre part, si les auteurs traitent parfois en termes quantitatifs de l'efficacité des techniques employées, il est rare que le souci d'éviter les artefacts apparaisse clairement comme l'une de leurs préoccupations majeures. Ceci est extrêmement regrettable car les rares publications dans lesquelles figure une étude critique des méthodes utilisées démontrent que les causes d'erreur sont multiples $[1,2,3]$.

Il n'est pas douteux que le fromage représente un milieu complexe (contenant en proportions sensiblement égales, eau, protéines et matière grasse) qui rend difficile l'extraction des composants de l'arôme. Ceci peut expliquer que de nombreux auteurs $[4,5,6]$ aient choisi d'étudier uniquement la phase grasse en postulant, d'après des critères organoleptiques, que celle-ci est entièrement représentative de l'arôme.

Comme à notre connaissance aucune étude objective n'a été publiée pour étayer cette hypothèse, nous avons pensé qu'il serait intéressant de comparer les profils chromatographiques obtenus en soumettant à la distillation sous vide poussé d'une part le fromage lui-même et d'autre part la matière grasse seule.

Par ailleurs, conscients que les procédés les plus élaborés ne sont pas toujours ceux qui donnent les résultats les meilleurs, il nous a semblé nécessaire de remettre en question certaines techniques en comparant la distillation sous vide poussé que nous avions précédemment utilisée [7] avec une méthode plus simple utilisant une technique de distillation sous reflux décrite par Forss et Holloway [8] et modifiée par Ramshaw [9]. 


\section{1) Extraction}

\section{MATERIEL ET METHODES}

Pour isoler les produits volatils du fromage nous avons utilisé deux techniques très différentes basées toutes deux sur la distillation sous vide de l'échantillon.

\section{a) Distillation sous vide poussé}

Cette expérience qui s'effectue sur une prise d'essai d'environ $100 \mathrm{~g}$ comporte deux temps. Dans un premier stade, l'échantillon de fromage (préalablement râpé et congelé) est soumis à un pompage dans les conditions précédemment décrites [7]. Cette opération qui dure de 8 à $10 \mathrm{~h}$ permet d'obtenir la quasi-totalité de l'eau présente dans le fromage ainsi que les substances les plus volatiles. Le lendemain, dans un second stade, le fromage (qui a été maintenu sous vide et placé au congélateur durant la nuit) est moulu très finement puis placé dans un ballon de 31 comportant un condenseur en doigt de gant décrit par Forss et Holloway [10]. L'ensemble est relié au groupe de pompage par l'intermédiaire d'une série de pièges (fig. 1). La très faible pression résiduelle régnant dans le système $(<10-4$ Torr $)$ ainsi que la faible distance séparant l'échantillon de la surface froide permettent de récupérer des substances extrêmement peu volatiles d'autant que le ballon est réchauffé par immersion dans un bain-marie maintenu à une température voisine de $30^{\circ} \mathrm{C}$ pendant toute la durée de l'expérience.

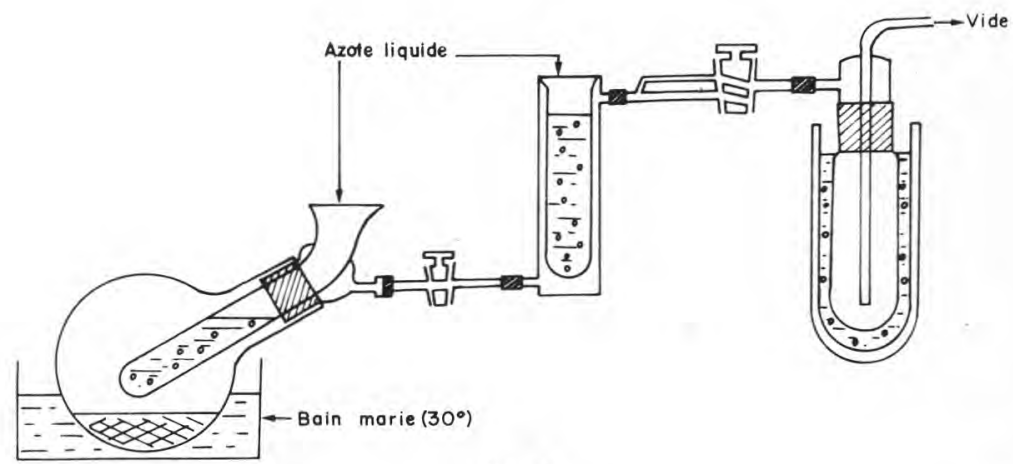

fig. 1

Montage utilisé pour obtenir les produits volatils à partir d'un échantillon pratiquement anhydre

b) Méthode de distillation sous reflux

Un échantillon de fromage de $100 \mathrm{~g}$ environ est coupé grossièrement en cubes puis, au moyen de l'ultra-turrax, réduit en quelques 
secondes à l'état de suspension par dispersion dans 250 à $300 \mathrm{ml}$ d'eau saturée par $\mathrm{NaCl}$. La suspension est transférée dans un ballon de 51 dans lequel on ajoute environ 11 d'eau saturée en $\mathrm{Na} \mathrm{Cl}$ ainsi qu'un barreau aimanté. Le ballon est alors connecté à un appareillage (fig. 2) comportant outre les réfrigérants et le ballon tampon (B) une série de pièges refroidis par l'azote liquide eux-mêmes reliés à une pompe à palettes qui permet d'abaisser la pression jusqu'à 0,1 Torr. On effectue le dégazage du système avec précaution, les pièges

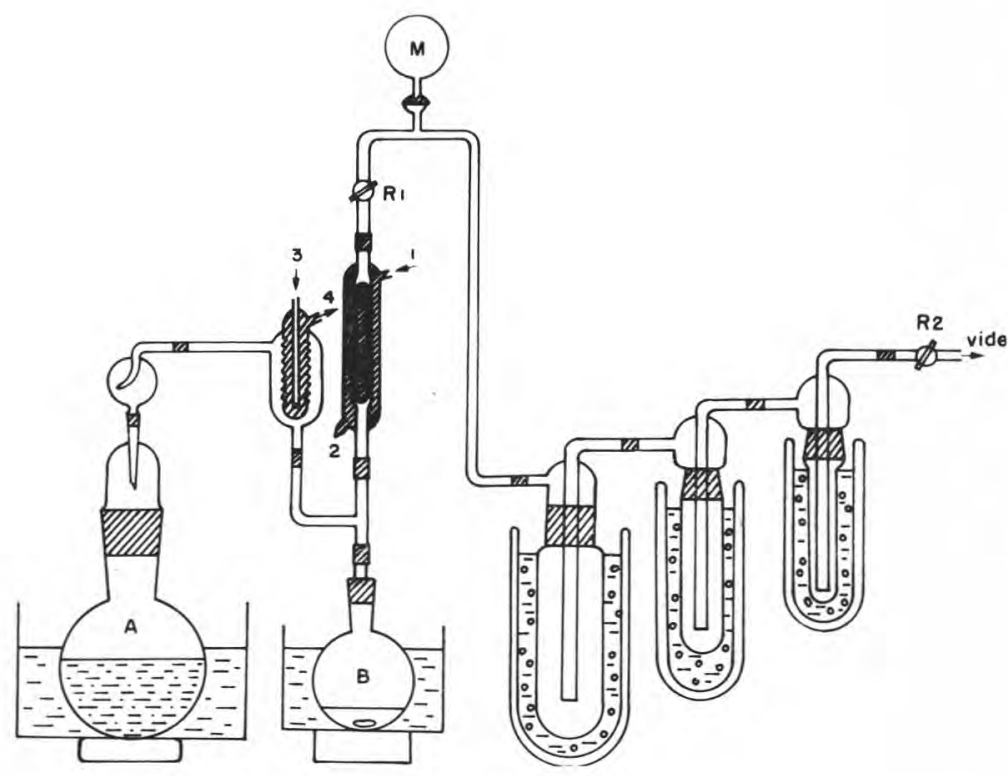

fig. 2

Dispositif servant à effectuer la distillation sous reflux des échantillons $(1,2,3,4)$ sens de circulation du fluide réfrigérant.

situés entre les 2 robinets faisant office de sas. Lorsque la pression résiduelle atteint quelques Torrs les agitateurs magnétiques sont mis en mouvement et la température de l'eau des bains-marie dans lesquels sont placés les ballons est portée à $40^{\circ} \mathrm{C}$, température qui n'est pas susceptible de provoquer des artefacts car l'expérience montre qu'il existe une différence de température de près de $20^{\circ} \mathrm{C}$ entre l'intérieur et l'extérieur des ballons (en raison de l'ébullition constante en A et des retours d'eau glacée condensée en B). La durée de pompage est de $8 \mathrm{~h}$ environ ; à la fin de cette période, près de 80 p. 100 de l'eau initialement présente dans le ballon A se retrouve dans le ballon $\mathrm{B}$. 
c) Cas où l'étude porte uniquement sur la matière grasse

Dans le cas où il s'agit d'apprécier la part de l'arôme revenant à la matière grasse, celle-ci est obtenue par centrifugation (centrifugeuse Servall, rotor SS $34,18000 \mathrm{t} / \mathrm{mn}, 50 \mathrm{mn}$ ) d'un échantillon de $100 \mathrm{~g}$ réparti dans des tubes vissés. La température de la cuve de la centrifugeuse est de $30^{\circ} \mathrm{C}$. On sépare ainsi la phase grasse avec des rendements de l'ordre de 65 à 70 p. 100 du maximum théorique.

La matière grasse ainsi obtenue est soumise à une distillation sous vide poussé avec le dispositif en doigt de gant. Le culot de centrifugation est dispersé à l'aide d'un Ultra-Turrax dans une solution de $\mathrm{NaCl}$ saturée puis soumis à une distillation sous reflux.

\section{d) Obtention des extraits}

Quelle que soit la méthode de distillation employée, on laisse fondre la glace contenue dans les pièges qui sont ensuite rincés très soigneusement avec de l'éther purifié. L'ensemble est collecté puis la phase aqueuse est saturée par $\mathrm{NaCl}$ et amenée à $\mathrm{pH} 9$ à l'aide d'une solution de soude normale. On procède alors à des extractions répétées au moyen de petites quantités d'éther ce qui conduit à la fraction neutre.

Dans le cas de la technique de distillation sous reflux le contenu des pièges et du ballon $\mathrm{B}$ sont traités séparément.

La phase aqueuse est ensuite acidifiée à $\mathrm{pH} 1$ par l'acide chlorhydrique normal et l'on procède à une nouvelle série d'extractions à l'éther : on obtient ainsi la fraction acide : Après séchage sur sulfate de sodium anhydre, la plus grande partie du solvant est éliminée par distillation sur des colonnes de Vigreux, ce qui permet de réduire les extraits à un volume de quelques centaines de $\mu 1$; ils sont alors prêts pour l'analyse par chromatographie en phase gazeuse.

\section{2) Analyse chromatographique}

On utilise un appareil Girdel (type 75-CD-PT) équipé de détecteurs à ionisation de flamme. L'extrait neutre est séparé sur une colonne en acier inox, longue de $3 \mathrm{~m}$ et d'un diamètre extérieur de 3,2 $\mathrm{mm}$ remplie de chromosorb G.AW.DMCS 60-80 mesh imprégné à 5 p. 100 par du Carbowax 20 M TPA (Applied Sciences) purifié tandis que dans le cas des extraits acides on utilise des colonnes longues de $2 \mathrm{~m}$ remplies de chromosorb W.AW.DMCS 80-100 mesh imprégné de 7,5 p. 100 d'adipate d'éthylène glycol et de 2 p. 100 d'acide phosphorique. La température du four augmente de façon linéaire à raison de $1,5^{\circ} \mathrm{C} / \mathrm{mn}$ de $50^{\circ} \mathrm{C}$ à $160^{\circ} \mathrm{C}$ pour l'analyse des produits neutres et de $75^{\circ} \mathrm{C}$ à $175^{\circ} \mathrm{C}$ pour celle des acides.

Afin de permettre une comparaison facile des chromatogrammes, le rapport pourcentage injecté de l'extrait total/atténuation du chromatographe a éte maintenu sensiblement constant. 


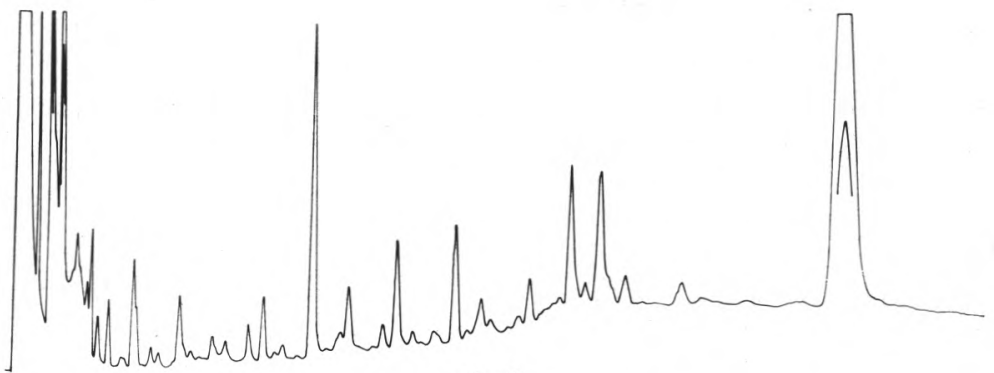

fig 3

Composés neutres obtenus par distillation sous vide poussé du fromage total

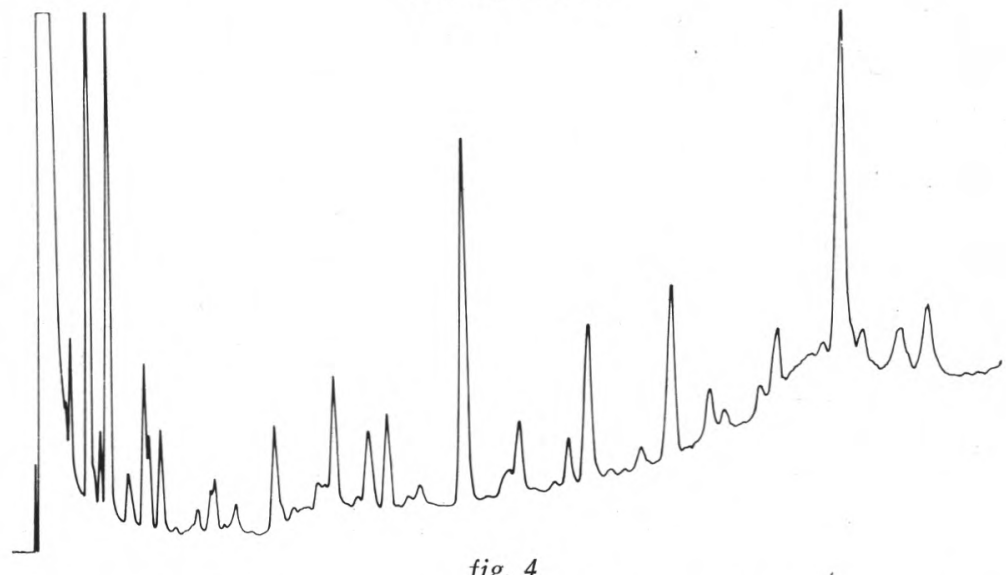

Composés neutres obtenus par distillation sous vide poussé de la matière grasse seule

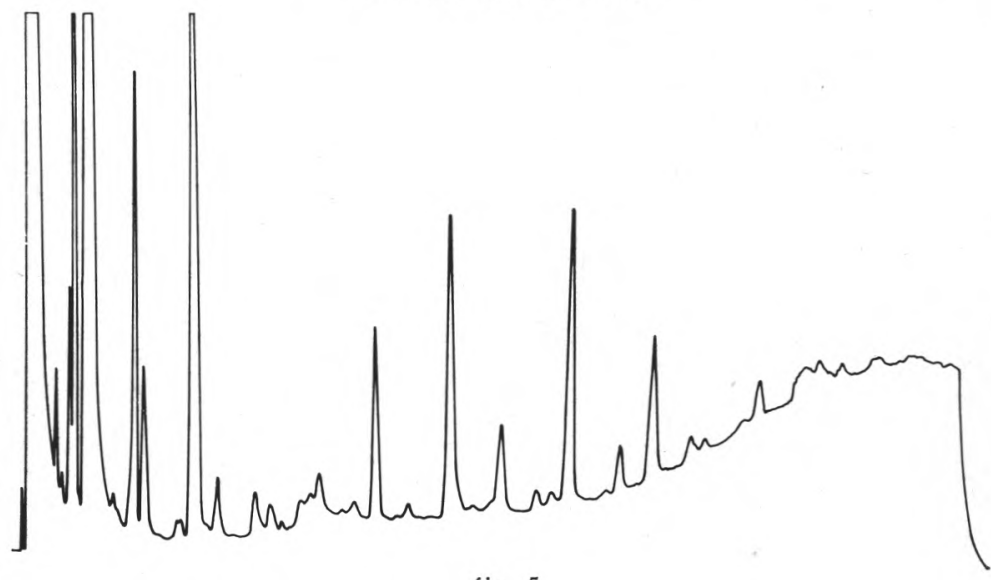

fig. 5

Composés neutres obtenus par distillation sous reflux du culot de centrifugation 


\section{RESULTATS ET DISCUSSION}

\section{1) Distribution des composants de l'arôme entre les différentes} phases du fromage

L'étude des chromatogrammes (fig. 3, 4 et 5) permet d'affirmer, que dans le cas des composés neutres, la corrélation entre les résultats obtenus avec le fromage total et ceux obtenus avec la matière grasse est excellente pour les produits de volatilité moyenne ; par contre pour les corps très volatils, la ressemblance est bien moins nette et le chromatogramme " complémentaire » obtenu par la méthode de distillation sous reflux laisse penser que bien des substances sont restées dans la phase aqueuse.

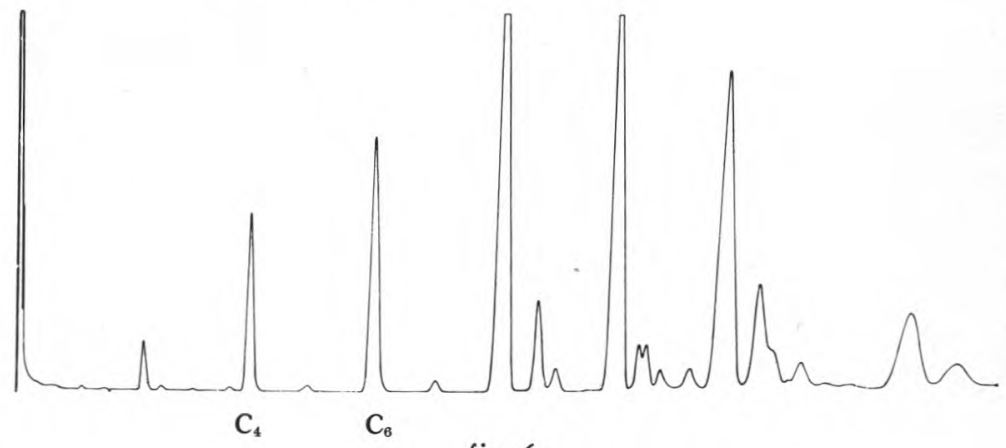

fig. 6

Composés acides provenant de la distillation sous vide poussé de

la matière grasse seule

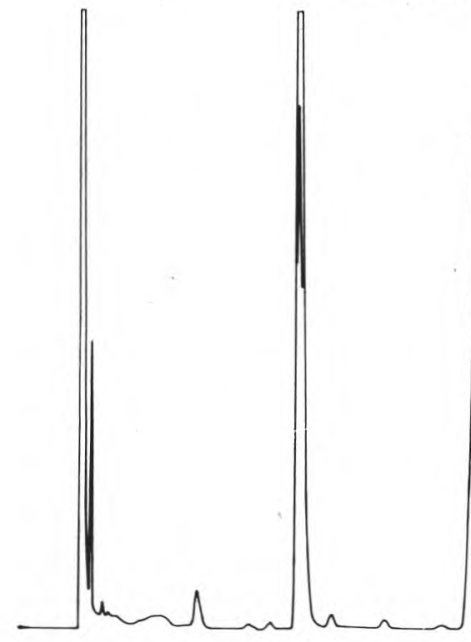

$\mathrm{C}_{4}$

\section{$\mathrm{C}_{\mathrm{B}}$
fig. 7}

Composés acides provenant de la distillation sous vide poussé du fromage total 
Pour les acides volatils la différence est très nette : les termes à courtes chaînes (jusqu'à l'acide en $\mathrm{C6}$ ), très solubles dans l'eau, sont pratiquement absents de la phase grasse (fig. 6) alors qu'ils sont présents en quantités importantes et en proportions similaires dans les extraits obtenus à partir du fromage total et du culot de centrifugation (fig. 7 et 8 ).

Ces essais permettent de penser que la conduite de l'expérimentation sur la seule matière grasse se justifie quand il s'agit d'obtenir certains composés peu volatils extrêmement difficiles à isoler [11]. Par contre, dans le cas d'une recherche globale sur les produits d'arôme cette méthode semble critiquable car elle provoque une sélection des corps en fonction de leur polarité.

Nous croyons qu'il serait sage de lui préférer une technique moins efficace mais qui respecte mieux les équilibres quantitatifs entre les différents constituants ou de la compléter par une méthode plus spécifique des composants les moins liposolubles.

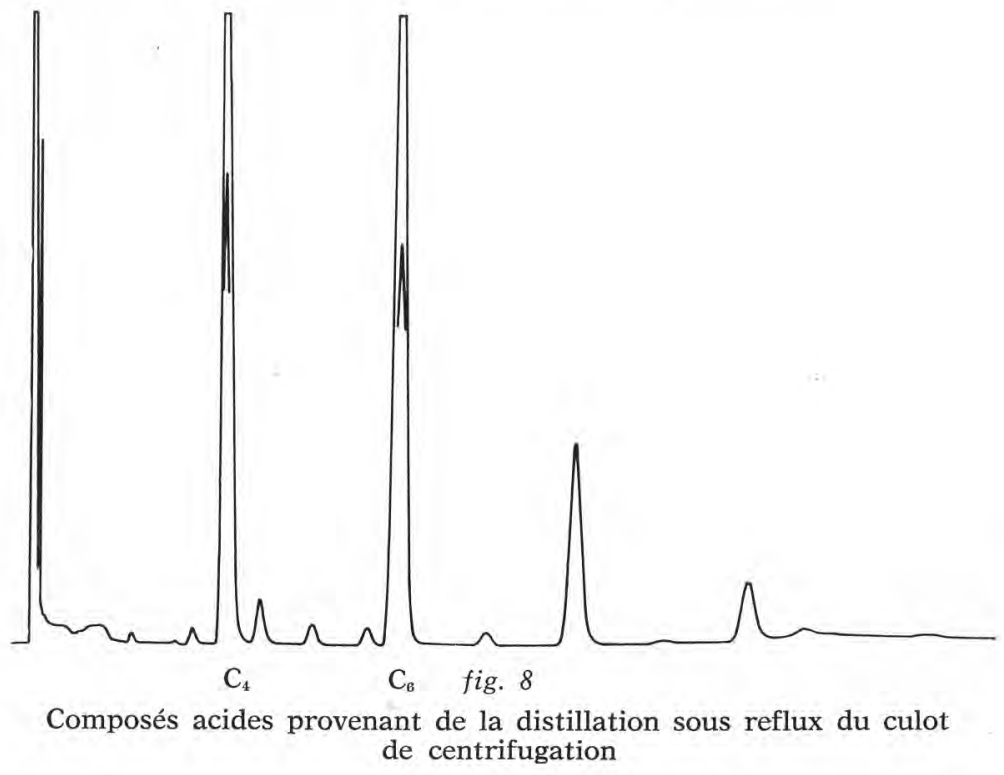

\section{2) Etude critique de la technique de distillation sous reflux}

Les résultats obtenus par l'application de cette technique à l'étude du culot de centrifugation nous ont semblé suffisamment probants pour nous amener à essayer de l'appliquer à une suspension préparée à l'aide d'un fromage non dégraissé. Nous avons donc, dans un premier temps, comparé les "profils » obtenus en soumettant une moitié d'un morceau de fromage à un pompage sous vide poussé et l'autre moitié à une distillation sous reflux. 
On a ensuite étudié la reproductibilité de la méthode en soumettant tour à tour les deux moitiés d'un même fromage à la technique de distillation sous reflux puis étudié l'efficacité de cette méthode en soumettant, après redilution dans 1,5 l d'eau saturée en $\mathrm{Na} \mathrm{Cl}$, le résidu présent dans le ballon $\mathrm{A}$ à une seconde journée de distillation.

Ces différents essais amènent à conclure :

a) qu'il y a pour les produits neutres, une corrélation étroite avec la distillation sous vide poussé du fromage total (fig. 9 et 10) tant au point de vue qualitatif qu'au point de vue quantitatif,

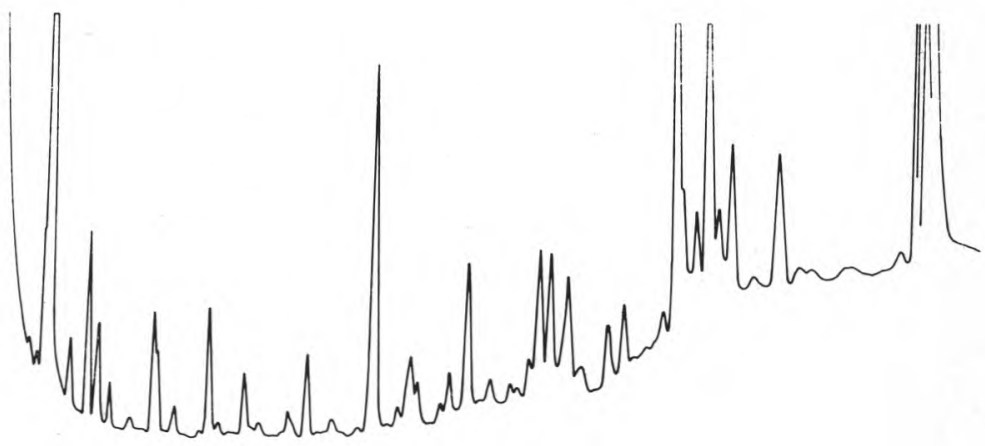

fig. 9

Composés neutres obtenus par distillation sous vide poussé d'une moitié du fromage $F_{1}$

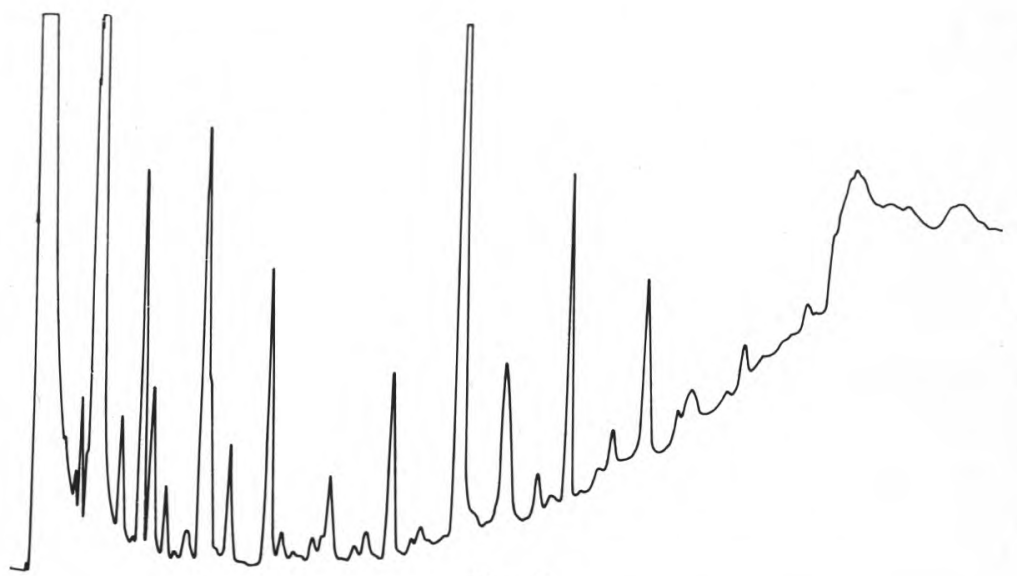

fig. 10

Composés neutres obtenus par distillation sous reflux de l'autre moitié du fromage $F_{1}$ 
b) que la méthode est reproductible puisque les 2 moitiés d'un même fromage traitées séparément fournissent des chromatogrammes analogues (fig. 11 et 12 ),

c) qu'elle est relativement efficace puisqu'un $2^{\text {me }}$ jour de distillation sous reflux n'augmente que fort peu la récupération des composés neutres (fig. 13). Cependant cela n'est pas vérifié pour les acides qui continuent à être présents en quantités importantes.

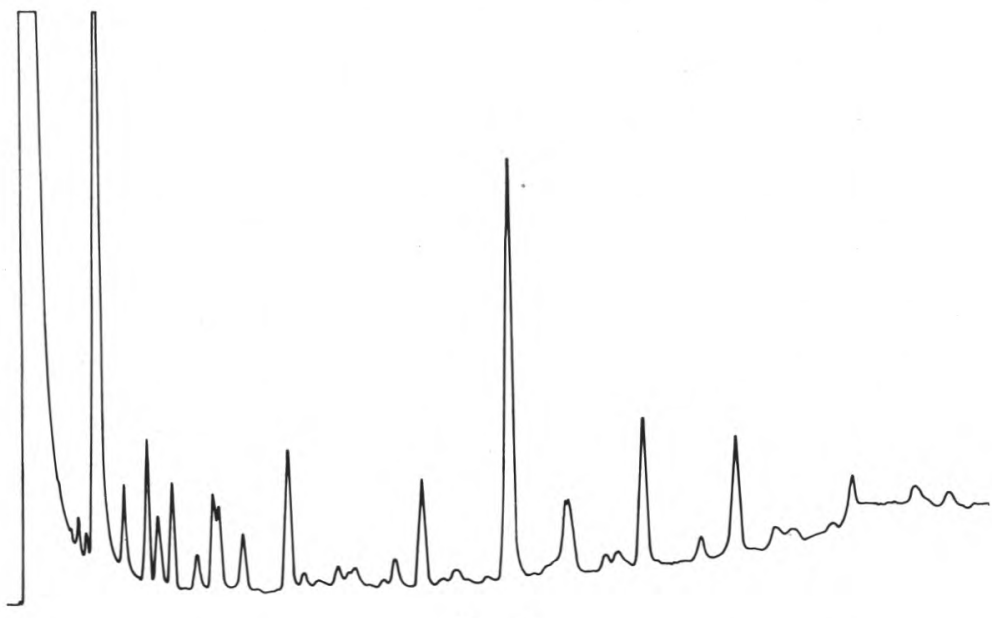

fig. 11

Composés neutres provenant de la distillation sous reflux d'une moitié $\mathrm{M}_{1}$ du fromage $\mathrm{F}_{2}$

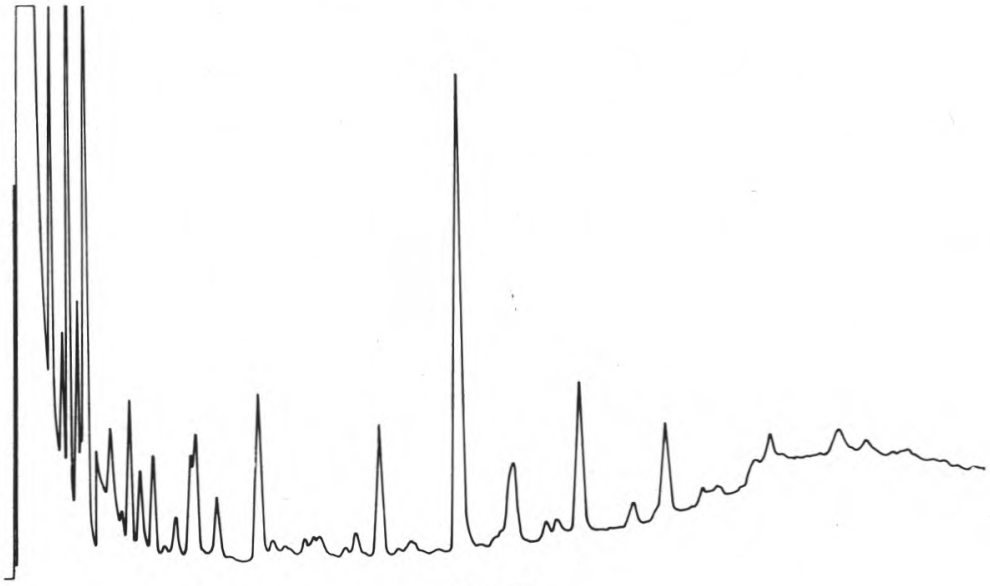

fig. 12

Composés neutres provenant de la distillation sous reflux de l'autre moitié $\mathrm{M}_{2}$ du fromage $\mathrm{F}_{2}$ 
Il faut signaler à ce propos que les composés neutres se trouvent en quasi-totalité dans les pièges tandis que les acides sont retenus dans le ballon "B ", ce qui simplifie le travail ultérieur d'extraction à l'éther.

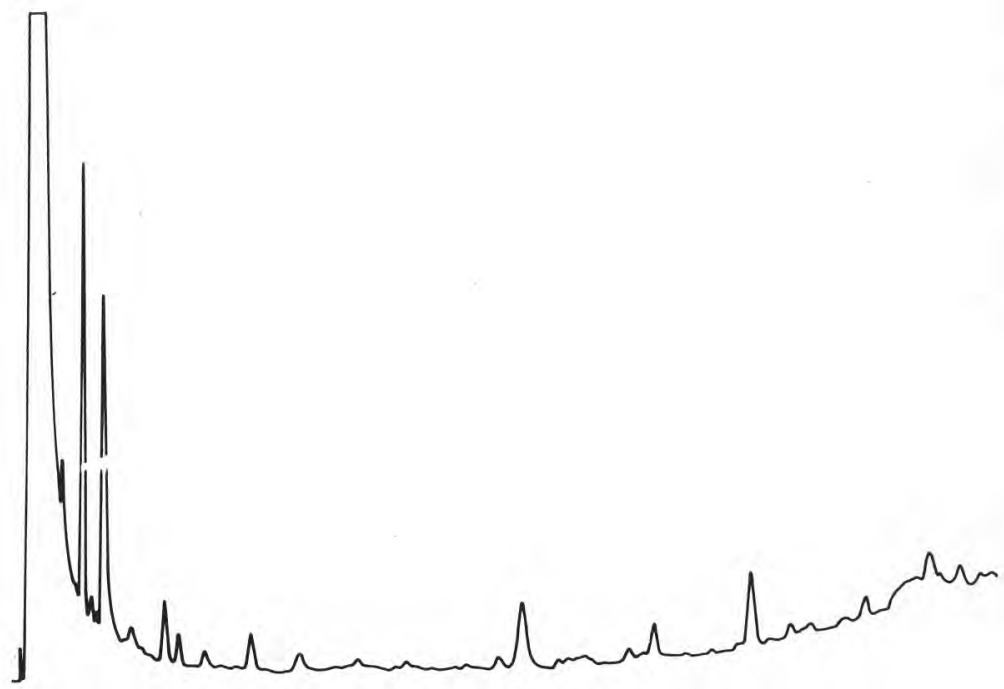

fig. 13

Composés neutres provenant d'une $2^{\text {me }}$ séance de distillation sous reflux de la moitié $\mathrm{M}_{2}$ du fromage $\mathrm{F}_{2}$

Enfin, pour compléter ce travail, nous avons déterminé expérimentalement quels pouvaient être avec cette technique les pourcentages de récupération d'un certain nombre de corps fréquemment impliqués dans l'arôme des fromages. A cet effet, nous avons ajouté des méthylcétones $\left(\mathrm{C}_{4}, \mathrm{C}_{5}, \mathrm{C}_{6}, \mathrm{C}_{\overline{7}}, \mathrm{C}_{7}, \mathrm{C}_{11}\right)$, et des alcools secondaires $\left(C_{4}, C_{5}, C_{6}\right)$ à un fromage préalablement soumis à 2 journées consécutives de distillation sous reflux et pouvant être considéré comme dépourvu d'arôme. La concentration finale des produits ajoutés avait été fixée à $1 \mathrm{ppm}$.

Parallèlement, afin de déterminer le rendement du stade « extraction par solvant - concentration ", nous avons traité un échantillon synthétique constitué par $10 \mathrm{ml}$ d'eau additionnée de la même quantité de substances témoins que le fromage.

Les résultats présentés dans le tableau 1, laissent apparaître des pourcentages de récupération voisins de ceux obtenus par d'autres auteurs $[4,8,10,12]$ qui utilisent des systèmes de pompages à vide poussé. 
TABLEAU 1

Pourcentages de récupération des composés témoins

\begin{tabular}{l|c|c|c}
\hline Substance & $\begin{array}{c}\text { p. 100 global de } \\
\text { récupération }\end{array}$ & $\begin{array}{c}\text { p. } 100 \text { de récupération pour le } \\
\text { stade extraction-concentration }\end{array}$ & $\begin{array}{c}\text { p. 100 de récupération pour le } \\
\text { stade «distillation sous reflux" }\end{array}$ \\
\hline & & & 55 \\
2 butanone & 37 & 80 & 58 \\
2 pentanone & 60 & 84 & 75 \\
2 hexanone & 61 & 84 & 73 \\
2 neptanone & 61 & 81 & 71 \\
2 undecanone & 48 & 78 & 62 \\
& & & 82 \\
2 butanol & 67 & 83 & 82 \\
2 pentanol & 70 & 86 & 85 \\
\hline
\end{tabular}




\section{CONCLUSION}

En fait, il n'y a vraisemblablement pas de méthode idéale susceptible de résoudre tous les problèmes. Cependant pour un type donné de recherches, certaines techniques se révèlent plus adéquates que d'autres. Ainsi la distillation sous vide poussé de la matière grasse peut être très féconde à condition de ne pas prétendre effectuer une étude globale.

Quant à la méthode de distillation sous reflux qui présente l'avantage de ne nécessiter qu'un matériel assez simple elle peut être avantageusement utilisée conjointement à l'étude des "Head Space » pour l'étude des composés relativement volatils et ce, même pour des travaux présentant un caractère de routine.

Reçu pour publication en décembre 1971.

\section{Ré s u m é}

Plusieurs méthodes d'isolement des composés volatils responsables de l'arôme d'un fromage ont été étudiées. Après avoir constaté l'insuffisance des méthodes fondées sur la séparation préalable de la matière grasse on compare une méthode d'extraction sous vide poussé à une méthode de distillation sous reflux. On étudie ensuite le pourcentage de récupération de produits purs ajoutés.

\section{S u m m a r y}

Several methods of extraction for cheese volatiles have been investigated. After showing that methods based on fat separation give poor recovery for the most volatile and (or) water soluble components, two differents methods are compared. One is a classical high vacuum distillation while the other is a distillation under reflux. Percentage recovery for various added pure compounds is given.

\section{Références bibliographiques}

[1] Weurman (C.), Groenen (P. J.) and Van Gemert (L. J.) (1970). - Experiments on "High-Vacuum Transfer " in Food Odour Research, Die Nahrung, 14, 7, 607.

[2] Weurman (C.) (1969). - J. Agr. Food. Chem., 17, 2, 370.

[3] KuRTs (F. E.). (1967). - J. Dairy Sci., 50, 6, 814. 
[4] Mc Gugan (W. A.) and Howsam (S. G.) (1962). - J. Dairy Sci., 45, 495.

[5] Liebich (H. M.), Douglas (D. R.), Bayer (E.) and Zlatkis (A.) (1970). J. of Chromatographic Sci., 8, 6, 351.

[6] Scarpellino (R.) and Kosikowski (F. V.) (1962). - J. Dairy Sci., 45, 495.

[7] Dumont (J. P.) et AdDA (J.) (1970). - Revue Lait. Franç., 279, 579.

[8] Forss (D. A.) JacoBsen (Valerie M.) and Ramshaw (E. M.) (1967). - J. Agr. Food. Chem., 15, 6, 1104.

[9] Ramshaw (E. M.). - (communication pers.).

[10] Forss (D. A.) and Holloway, Gwenda (L.) (1967). - J. Am. Oil. Chem. Soc., $44,10,572$.

[11] Forss (D. A.) Urbach Gerda and StaRK (W.) (1966). - XVII Congr. Intern. Lait Munich, C, 2, 211.

[12] Nakanishi (T.) and Arai (I.) (1970). - Jap. J. Dairy Sci., 19, 6 A 169-A 175. 